\title{
Prevalence rate of Klebsiella pneumoniae in the intensive care unit: epidemiology and molecular characteristics
}

\author{
Soad A. Abdallah, Sanaa S. Zaki, Safa S. Hafez, Elham E. Moustafa \\ Department of Botany, Faculty of Women for Arts, Science and Education, Ain Shams University, Cairo, Egypt
}

\begin{abstract}
Klebsiella pneumoniae carbapenemase producing bacteria are defined as a group of Gram-negative bacilli that are highly resistant to drugs. The resistance of pathogens of the Enterobacteriaceae family to $\beta$-lactam antibiotics such as carbapenems is considered a major threat in the medical field. The main aim of the current study was to explore and confirm the occurrence of carbapenemase producing $K$. pneumoniae in the Intensive Care Unit (ICU) in different hospital environment sample sites of two Egyptian hospitals in Cairo. Isolates were collected and the screening criteria of carbapenemase producing bacteria
\end{abstract}

Correspondence: Soad A. Abdallah, Department of Botany, Faculty of Women for Arts, Science and Education, Asmaa Fahmy Street, Heliopolis, Ain Shams University, 11757 Cairo, Egypt.

Tel.: 002.01204655989 - Fax: 002.02.24157804.

E-mail: husseinsoad@gmail.com

Key words: Intensive care unit pathogens; Inanimate sources; Antibiotic resistant Klebsiella pneumoniae; Carbapenemase.

Contributions: the authors contributed equally.

Conflict of interest: the authors declare no potential conflict of interest.

Funding: financial support by the Public Administration of Environmental Projects - Applied Research Committee, Community Service and Environmental Development Sector, Ain Shams University, Grant no. 1-2017.

Acknowledgements: the authors would like to thank Dr. Manal Farouk M. Abdelall, Department of Microbial Molecular Biology, Agricultural Genetic Engineering Research Institute (AGERI), Agricultural Research Center (ARC), for her help and support. The authors would also like to thank Dr. Enas H. Ghallab, Faculty of Science, Ain Shams University for her helpful efforts and time.

Received for publication: 22 March 2018.

Revision received: 7 June 2018.

Accepted for publication: 8 June 2018 .

(C) Copyright S.A. Abdallah et al., 2018

Licensee PAGEPress, Italy

Journal of Biological Research 2018; $91: 7437$

doi:10.4081/jbr.2018.7437

This article is distributed under the terms of the Creative Commons Attribution Noncommercial License (by-nc 4.0) which permits any noncommercial use, distribution, and reproduction in any medium, provided the original author(s) and source are credited. was followed by the investigators in order to record the antimicrobial resistance patterns of all isolates in addition to the molecular identification using the $16 \mathrm{~S}$ rDNA.

This study determined the sites responsible for the spreading of carbapenem-resistant $K$. pneumoniae including suction tubes, ventilator tubes, instrument tables and beds. All K. pneumoniae isolates collected from the ICU of both hospitals were resistant to oxacillin, meropenem and ceftazidime. Moreover, 16S rDNA gene sequence was used to study bacterial phylogeny and taxonomy for all $K$. pneumoniae isolates; the accession number of all isolates is reported. We concluded that infection control department policies in each hospital should be reinforced to avoid the escalation of $K$. pneumoniae as nosocomial infections in hospitals. This study should be repeated in other hospitals (especially the public hospitals) to assess the level of the problem.

\section{Introduction}

Klebsiella pneumoniae carbapenemase (KPC) producing bacteria is a group of Gram-negative bacilli that are highly resistant to drugs and cause lethal infections and illnesses. ${ }^{1,2}$ Their first isolation was in the north-eastern part of the United States. The first detection of KPC took place more than ten years ago. ${ }^{3}$ The infections that are caused by the KPC producing bacteria are a serious problem discussed all over the world. ${ }^{4}$ The incidence of KPC is increasing at a worrisome rate due to variable reasons since the treatment of these infections is very challenging due to limited options of antibiotics that fight these bacteria. ${ }^{5,6}$ Moreover, these infections are associated with higher mortality rate, long hospital stay duration, and an increasing cost of hospital care. ${ }^{7}$ Despite the fact that they are not the only carbapenem resistant bacteria, KPCs require a unique mechanism of detection other than the routine susceptibility screening which proved to be insufficient for the detection of KPCs. ${ }^{8}$ The efficiency of carbapenems lies in their ability to treat infections that are caused by multi-drug resistant Gram-negative bacteria. These therapeutic agents, however, are constrained by the emergence of resistant isolates, which affect their efficacy to treat these infections. When isolates produce extended-spectrum $\beta$ lactamases (ESBLs), carbapenems are used in hospitals to treat infections caused by K. pneumoniae. Resistance to carbapenems is still infrequent in Enterobacteriaceae even though it is common among Gram-negative non-fermenting bacteria. ${ }^{9}$ The detection of carbapenem-resistant $K$. pneumoniae is aggregating in different areas all over the world in which carbapenem resistance is mainly caused by KPC enzymes or where metallo- $\beta$-lactamases (MBLs) dominate. ${ }^{10-12}$ According to Livermore, MBLs of the VIM type are more common among nonfermenting gram-negative bacteria. ${ }^{13}$ Recently, studies have confirmed the diffusion of VIM-type MBLs 
in members of the family Enterobacteriaceae. ${ }^{14}$ This explains the continuing spread of these resistance determinants among pathogens with higher contamination. ${ }^{15}$ This is the first prospective study in Egypt that examines the environmental contributions rather than patients. This study will not tackle isolates collected from patients since this point has sufficiently been addressed in previous studies conducted in Egypt and other countries. The study, however, focuses on inanimate environmental sites in Intensive Care Units (ICUs) as another source of nosocomial infection. The main aim of the current study is to explore and confirm the occurrence of carbapenemase producing $K$. pneumoniae in the ICU's of hospital environment sample sites of two Egyptian hospitals in Cairo. At the end of the study, the investigators will determine the sites responsible for the spreading of spreading of carbapenem-resistant K. pneumoniae.

\section{Materials and Methods}

\section{Sample collection}

Samples were collected from the ICU of two hospitals which will be referred to as $\mathrm{N}$ and $\mathrm{Z}$ for confidentiality; the two letters $\mathrm{N}$ and $\mathrm{Z}$ are the initials of the hospital names. The two hospitals are located in Cairo, Egypt. The ICU of hospital $\mathrm{N}$ has four different rooms: the main ICU, medical surgical, the extension and the source isolation room, in addition to the instrument store that includes all instruments and tools used when needed. Triplicate swabs were collected from each compartment of the rooms and stores mentioned above including: air conditioning, walls next to patients' beds, drug cabinets, sink tabs, windows, ICU doors (front and back), patients' beds, air suctions, curtains, under bed floor, surface of the located instruments, switches, patients' file trays, nurses clothing, patients clothing, located table contaminated by dry blood, laundry patients clothing boxes, instruments' trays and food tables. Swabs were also collected from suction tubes, ventilator tubes, oxygen instrument holder, ventilator tubes that are attached to patients, area located around the ventilator, nasal tubes attached to patients, nasal tubes, tubes attached to dialysis, air bedding of patients beds, patients tongue depressor, and vacuum hole as well as oxygen hole located on the wall. The ICU of hospital Z has only the ICU of internal medicine. Triplicate swabs were collected from different hospital environment sample sites including washed suction tube, covers and tubes suction used internally for patients, nasal secretion from suction, blood contaminated suction, ventilator surface area, rubbish bin cupboard, oxygen holder attached to a patient, the wall of suction tube, counters, taps and sinks, ambo connected to patients, curtains, shelves of a cupboard, solutions tray, below the sink, syringes holder, patients beds, used syringes bins, file desks, ventilator tubes connected to patient, windows, curtains with dry blood, ultrasonic gel containers, the water of ventilator and the steel parts of beds.

\section{Microbiological studies}

The collected swabs were transferred immediately to the lab for isolation and purification of $K$. pneumoniae. For isolation of the microorganism, Oxoid MacConkey agar plates (Oxoid LTD, Basingstoke, Hampshire, England) were used; the pink mucoid colonies were purified on MacConkey agar plates. For the identification of the pure suspected $K$. pneumoniae, chromagar KPC media (CHROMagar Paris-France) was used that gives metallic blue colonies. The suspected $K$. pneumoniae isolates underwent urease activity, motility test, growth on citrate agar medium, growth on triple sugar iron, methyl red test, and growth on SIM medium (Tryptone $20.0 \mathrm{gm} / \mathrm{L}$, Peptone $6.1 \mathrm{gm} / \mathrm{L}$, Ferrous ammonium sulphate $0.2 \mathrm{gm} / \mathrm{L}$, Sodium thiosulphate $0.2 \mathrm{gm} / \mathrm{L}$, Agar 3.5 $\mathrm{gm} / \mathrm{L}$ ), in addition to nitrate reduction test. For final confirmation, API 20E test (BIOMERIEUX, Marcy l'Etoile, France) was used and resulted in the identification of 13 specimens of KPC.

\section{Antimicrobial susceptibility testing}

Susceptibility testing was performed using disk diffusion method on Muller-Hinton agar plate (Oxoid LTD, Basingstoke, Hampshire, England) with the antibiotic discs according to Clinical and Laboratory Standards Institute guidelines. Antibiotics tested comprise: imipenem $(10 \mu \mathrm{g})$, meropenem $(10 \mu \mathrm{g})$, tigecycline $(15 \mu \mathrm{g})$, oxacillin $(1 \mu \mathrm{g})$, sulphamethoxazole trimethoprime $(25 \mu \mathrm{g})$, chloramphenicol $(10 \mu \mathrm{g})$ (from Oxoid), and ceftazidime $(30 \mu \mathrm{g})$, gentamicine $(30 \mu \mathrm{g})$ (from Bioanalysis).

\section{Identification of the bacterial isolate $16 \mathrm{~S}$ rDNA}

The selected bacterial isolates were identified using 16S rDNA sequencing as molecular tools. Genomic DNA from each of the bacterial colonies was isolated as described by Sambrook et al. ${ }^{16}$ Amplification of the 16S rDNA region from the extracted DNA of bacterial isolates was performed using polymerase chain reaction (PCR), oligonucleotide primers pair, 27F 5'AGAGTTTGATCMTGGCTCAG -3'and 1492R 5'-CGGTTACCTTGTTACGACTT-3'. Each reaction contains $14.5 \mu \mathrm{L}$ of deionized sterile water, $2.5 \mu \mathrm{L}$ of buffer $10 \mathrm{X}, 1 \mu \mathrm{L}$ of $\mathrm{MgCl} 2(50 \mathrm{mM})$, $0.5 \mu \mathrm{L}$ of dNTP's $(20 \mathrm{mM}), 2 \mu \mathrm{L}$ of each primer $(10 \mathrm{pM}), 0.5 \mu \mathrm{L}$ of Taq polymerase $(5 \mathrm{U} / \mathrm{mL})$ and $2 \mu \mathrm{L}$ of sample DNA. PCR amplification was carried out under the following conditions: initial denaturation $94^{\circ} \mathrm{C}$ for 5 min followed by 35 cycles of denaturation at $94^{\circ} \mathrm{C}$ for 1 minute, annealing $65^{\circ} \mathrm{C}$ for 1 minute and elongation at $72^{\circ} \mathrm{C}$ for 1 minute and final extension at $72^{\circ} \mathrm{C}$ for $7 \mathrm{~min}$. The amplified products were separated by electrophoresis on $1.0 \%$ agarose gel stained with ethidium bromide $(0.5 \mu \mathrm{g} / \mathrm{mL})$ and was visualized on UV gel documentation system (BioRad, USA). PCR products were purified from unincorporated PCR primers and dNTPs using QIAquick PCR Purification Kit (Qiagen, Netherlands), following the manufacturer's instructions. The $16 \mathrm{~S}$ rDNA reference sequences were BLAST searched on the National Center for Biotechnology Information (NCBI) database and compared with the obtained sequence on the Sequence ${ }^{\circledR}$ Software. Sequencing runs were performed at LGC Genomics $\mathrm{GmbH}$ on a 3730xl DNA Analyzer (Applied Biosystems ${ }^{\mathrm{TM}} /$ Thermo Fisher Scientific). DNA sequences obtained in this study were submitted to the NCBI database (accession No. MG461513- MG461525).

\section{Sequence alignments and phylogenetic analysis}

After PCR purification, the nucleotide sequences of the 13 specimens confirmed through the above-mentioned microbiological tests were sequenced in both directions. The obtained sequences from our specimens as well as the sequences downloaded from GenBank for other strains of the same species, all were initially aligned with CLUSTAL Omega method. The aligned sequences were saved as fasta files that have been used as a matrix to estimate the phylogeny of the entire alignments by constructing Neighbor-Joining (NJ) tree in MEGA 7 Software. ${ }^{17}$ The phylogenetic tree was constructed and performed with 1000 bootstrap replications to evaluate the reliability of the constructions. The evolutionary distances were computed using the p-distance method and are in the units of the number of base differences per site. The 
analysis involved 18 nucleotide sequences. Codon positions included were $1^{\text {st }}+2^{\text {nd }}+3^{\text {rd }}+$ Noncoding. All positions containing gaps and missing data were eliminated.

\section{Results}

From all the biochemical tests mentioned above 13 isolates of K. pneumoniae were confirmed. Among them seven isolates (54\%) were collected from hospital $\mathrm{N}$ and six (46\%) from hospital Z. $K$. pneumoniae from hospital $\mathrm{N}$ were isolated only from the main ICU and the source isolation room of hospital environment sample sites including instrument's table, ventilator tube, food table, patient's bed from the main ICU, while K. pneumoniae were collected from the source isolation room from suction tube, oxygen tube holder and patient tongue depressor.

On the other hand, the isolates collected from ICU hospital environment sample sites of hospital $\mathrm{Z}$ were two isolates from the suction tubes, and one isolate from the surface of the suction, ventilator tube, patient's bed and the suction mask.

The thirteen $K$. pneumoniae isolates collected from the hospital environment sample sites of the ICU of both hospitals were resistant to oxacillin, meropenem and ceftazidime. Nine isolates (69\%) were resistant to chloramphenicol and sulphamethoxazole trimethoprim, and four isolates $(31 \%)$ collected from the ventilator tube, the two suction tubes and suction surface of the ICU of hospital $\mathrm{Z}$ were sensitive to both antibiotics. Six isolates (46\%) were resistant to gentamicin, while seven (54\%) were resistant and were isolated from instrument's tables of the ICU of hospital N, and from the suction tube and its surface. It is worth noting that 11 isolates (85\%) were sensitive to imipenem and tigecycline as shown in Tables 1 and 2. A total of $85 \%$ of $K$. pneumoniae isolates were ESBL producers.

Previous studies suggested that $16 \mathrm{~S}$ rDNA sequencing is considered an accurate method for species identification and distinguishing between closely related bacterial species. The selected isolates were further analyzed by $16 \mathrm{~S}$ rDNA sequencing and submitted to GenBank with the accession numbers MG461513, MG461514, MG461515, MG461516, MG461517, MG461518, MG461519, MG461520, MG461521, MG461522, MG461523, MG461524 and MG461525. The phylogenetic analyses of their $16 \mathrm{~S}$ rDNA sequences according to the available data are summarized in Table 3 and Figure 1.

Table 1. Antibacterial susceptibility test for Klebsiella pneumoniae isolates. Average diameter was taken for each inhibition zone.

\begin{tabular}{|c|c|c|c|c|c|c|c|c|}
\hline $\begin{array}{l}\text { Klebsiella } \\
\text { pneumoniae } \\
\text { Isolates } \\
\text { and Codes }\end{array}$ & $\begin{array}{c}\text { Chloramphenicol } \\
\text { (30 } \mu \mathrm{g})\end{array}$ & $\begin{array}{c}\text { Sulphamethoxazole } \\
\text { trimethoprim } \\
\text { (25 jg) }\end{array}$ & $\begin{array}{r}\text { Inhi } \\
\text { A } \\
\text { Oxacillin } \\
(1 \mu g)\end{array}$ & $\begin{array}{l}\text { bition Zone (1 } \\
\text { tibiotic Agen } \\
\text { Gentamicin } \\
(10 \mu \mathrm{g})\end{array}$ & $\begin{array}{l}\mathrm{nm}) \\
\text { ts } \\
\text { Ceftazidime } \\
\quad(30 \mu \mathrm{g})\end{array}$ & $\begin{array}{l}\text { Tigecycline } \\
\text { (15 } \mu \mathrm{g})\end{array}$ & $\begin{array}{l}\text { Meropenem } \\
\text { (10 jg) }\end{array}$ & $\begin{array}{c}\text { Imipenem } \\
\text { (10 jg) }\end{array}$ \\
\hline 1 (Nla) & - & - & - & 15.5 & - & 11 & - & 21.5 \\
\hline 2 (N1b) & - & 22 & - & - & - & 9 & - & - \\
\hline 3 (N1a') & - & 8 & - & - & - & 12 & - & 12 \\
\hline 4 (Nlc) & - & - & - & - & - & 9 & - & 9 \\
\hline 5 (N2d) & - & - & - & - & - & 10 & - & 12 \\
\hline $6(\mathrm{~N} 2 \mathrm{e})$ & - & - & - & 17 & - & 12 & - & 24.5 \\
\hline 7 (N2f) & - & - & - & - & - & 11 & - & 13.5 \\
\hline 8 (Z1d) & 14.5 & 16 & - & 16.5 & - & 11 & - & 23 \\
\hline 9 (Z1d') & 20 & 18.5 & - & 17 & - & 11 & - & 25 \\
\hline 10 (Z1d") & 21 & - & - & 16 & - & 10 & - & 25.5 \\
\hline 11 (Z1b') & - & - & - & - & - & 10.5 & - & 8.5 \\
\hline 12 (Z1c') & - & - & - & 11.5 & - & - & - & 2.2 \\
\hline 13 (Z1d"”) & 22 & 15.5 & - & - & - & 10.5 & - & - \\
\hline
\end{tabular}

Table 2. Interpretation of the in vitro antibiotic susceptibility test results.

\begin{tabular}{|c|c|c|c|c|}
\hline Antibiotic Agent & Disc Concentration & Susceptible (S)(mm) & Susceptible-dose dependent (mm) & Resistant (R) (mm) \\
\hline Impenem & $10 \mu g$ & $\geq 18$ & $15-17$ & $\leq 14$ \\
\hline Meropenem & $10 \mu g$ & $\geq 16$ & $14-15$ & $\leq 13$ \\
\hline Tigecycline & $15 \mu g$ & $\geq 19$ & $9-12$ & $\leq 0.002$ \\
\hline Ceftazidime & $30 \mu g$ & $\geq 18$ & $15-17$ & $\leq 14$ \\
\hline Gentamycin & $10 \mu g$ & $\geq 15$ & $13-14$ & $\leq 12$ \\
\hline Oxacillin & $1 \mu g$ & $\geq 15$ & 14 & $\leq 13$ \\
\hline Sulphamethoxazole trimethoprime & $25 \mu g$ & $\geq 16$ & $11-15$ & $\leq 10$ \\
\hline Chloramphenicol & $30 \mu g$ & $\geq 18$ & $13-17$ & $\leq 12$ \\
\hline
\end{tabular}




\section{Phylogenetic analysis}

Phylogenetic relationships based on the aligned $16 \mathrm{~S}$ sequences were performed to analyse the genetic divergence among K. pneumoniae investigated in this study. NJ tree constructed showed four major distinguished clades (Figure 2).

MG461514.1, MG461515.1, MG461516.1, MG461518.1 and MG46153.1 from $K$. pneumoniae, fall in one clade with KR269806.1, KU724411.1 and MG551533.1. There was no sequence difference enough to separate them.

MG551533.1, MG461521.1 and MG461513.1 form a monophyletic group as G551533.1 have common ancestor relationship with MG461521.1 and MG461513.1, which both are sister groups to each other. The same was shown with KX170832.1 that has common ancestor relationship with MG461524.1 and MG461522.1, both are sister groups to each other. Finally, MG461520.1, MG461519.1, MG461517.1 and MG461525.1 formed a single clade.

\section{Discussion}

This study tackles the hospital environment sample sites in the ICU of two hospitals in the city of Cairo. Previous literature collected samples from patients in the ICU; this study, however, is the first in the Middle East and North Africa region to focus on inanimate hospital environment sample sites instead of patients. As microbiologists, our main goal was to isolate $K$. pneumoniae carbapenemase, which have high resistance to antimicrobial drugs. This resistance is rapidly increasing in comparison with the development of antimicrobial drugs, which is slow-paced. Resistant bacteria are a major threat to the economy of the country as well as our healthcare sectors. ${ }^{18,19}$ In the healthcare setting, Carbapenemresistant (CRE) organisms are major infectious agents and are believed to cause high rates of mortality amongst critical cases in hospitals and patients who are hospitalized for a long period and are exposed to dangerously severe procedures. ${ }^{20}$ The results of the current study reveal that K. pneumoniae was found and isolated from various ICU hospital environment sample sites including ventilator tubes, oxygen holders, beds, the suction tube and even instrument tables. The phylogenetic analysis of $16 \mathrm{~S}$ rDNA, as shown in the results section of this study, helped identify and classify $K$. pneumoniae. It is worth noting that it could be used to con-

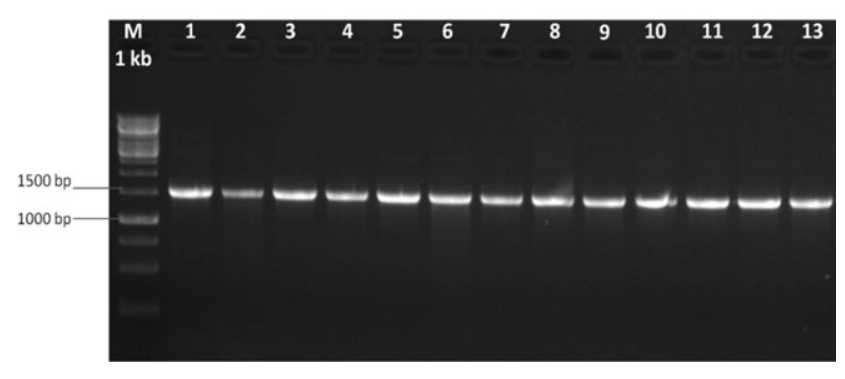

Figure 1. Agarose gel electrophoresis of Amplified polymerase chain reaction products for $16 \mathrm{~S}$ amplicons from 13 bacterial isolates on $1.0 \%$ agarose gel stained with ethidium bromide. M 1 kb ladder.

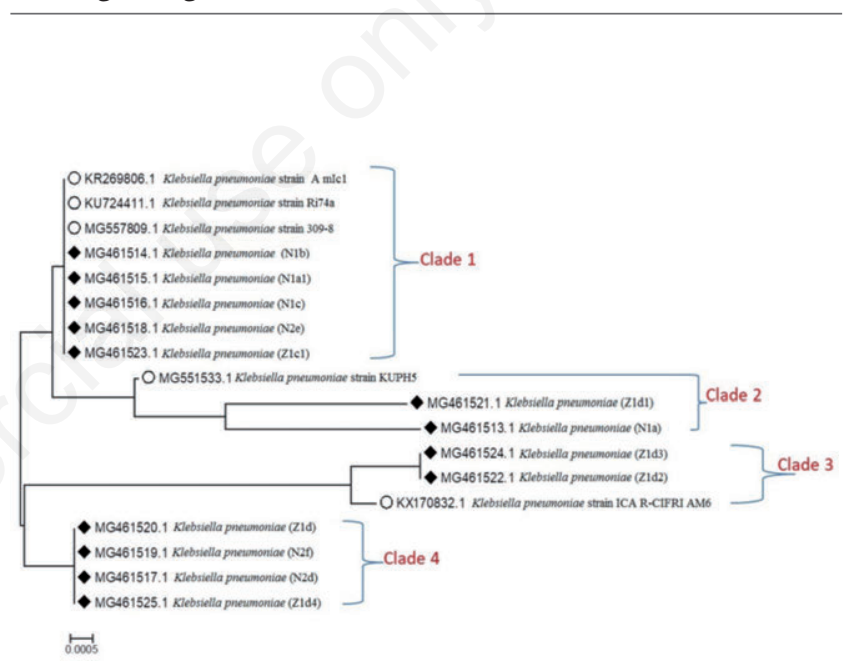

Figure 2. The phylogenetic tree of Klebsiella pneumoniae isolates.

Table 3. GenBank accession number(s) for 16S Klebsiella pneumoniae isolates nucleotide sequence(s).

\begin{tabular}{lccc}
$\begin{array}{l}\text { Isolates accession number and their sequence similarities based on 16SrDNA } \\
\text { Isolates (Sequence ID) }\end{array}$ & $\begin{array}{c}\text { Source } \\
\text { Accession number }\end{array}$ & 99\% similarity strains \\
N1a & MG461513 & Hospital (1) (instrument table) & Klebsiella pneumoniae strain F3-1-28 \\
N1b & MG461514 & Hospital (1) (ventillator tube) & K. pneumoniae strain QS17-0161 \\
\hline N1al & MG461515 & Hospital (1) (instrument and food table) & K. pneumoniae isolate AHII-3 \\
N1c & MG461516 & Hospital (1) (patient bed) & K. pneumoniae strain 309-8 \\
\hline N2d & MG461517 & Hospital (1) (suction tube) & K. pneumoniae strain Amlc1 \\
N2e & MG461518 & Hospital (1) (tongue depressor) & K. pneumoniae strain QS17-0161 \\
\hline N2f & MG461519 & Hospital (1) (oxygen mask) & K. pneumoniae strain 309-8 \\
Z1d & MG461520 & Hospital (2) (suction tube) & K. pneumoniae strain QS17-0161 \\
\hline Z1d1 & MG461521 & Hospital (2) (suction tube) & K. pneumoniae strain KUPH5 \\
Z1d2 & MG461522 & Hospital (2) (suction surface) & K. pneumoniae strain 309-8 \\
\hline Z1c1 & MG461523 & Hospital (2) (patient bed) & K. pneumoniae strain ATCC 700603 \\
Z1d3 & Hospital (2) (suction mask) & K. pneumoniae strain ICAR-CIFRI AM6 \\
\hline Z1d4 & MG461524 & Kospital (2) (suction mask) & Kneumoniae strain $309-8$ \\
\hline
\end{tabular}


firm subsp. Pneumonia, but could not separate other subspecies of K. pneumoniae completely. ${ }^{21}$

Previous studies also revealed similar existence in humidifiers of incubators, biofilms, sinks, sewage systems, surfaces such as beds, fabrics including linen, curtains and pillows, computer keyboards and hospital food utensils. ${ }^{22-26}$ Gram negative bacteria are mostly found in humid and moist settings. So they are typically found in liquid soap, pools, water fountains, infusion pumps, cleaning mops and respiratory equipment. ${ }^{27,28}$ Yet, a systematic examination of inanimate surfaces revealed that Gram-negative species, such as Acinetobacter spp., and Klebsiella spp, have the ability to survive on inanimate and dry surfaces for a long period of time (for months). ${ }^{29}$

Conversely, according to Weber et al., less than $15 \%$ of CRE found on immunized surfaces in patient rooms can survive for 24 hours and with low levels of contamination $\left(5.1 \mathrm{cfu} / 120 \mathrm{~cm}^{2}\right){ }^{30,31}$ However, more studies are needed to generalize the findings of the previous study to be able to conclude that the risk of the transmission of CRE from environmental surfaces is comparatively low. Additionally, the transmission of CRE from all types of surfaces should be considered in future studies. ${ }^{32}$

The present study shows that $100 \%$ of $K$. pneumoniae isolates were resistant to oxacillin, meropenem and ceftazidime. Similar results were found in other studies which also indicated that Multi Drug Resistant (MDR) organisms have been found in several cases in ICU settings. ${ }^{2}$ Appropriate infection control strategies have been implemented in many ICUs where MDR organisms have been isolated, as well as standard infection control practices including hand hygiene before and after unit entry as well as routine disinfection and cleaning techniques and strategies such as sterile techniques when inserting the catheters and maintaining unhindered urine drainage. ${ }^{33,34}$ In some healthcare centres, it is advisable to use periodic screening surveillance cultures to detect resistant pathogens in the ICU. ${ }^{35}$ In addition to the hygienic sanitary facilities such as rimless toilets, appropriate sink and faucet design such as sloped angles to reduce splashing. Moreover, a self-disinfecting sink has proven to be successful at reducing the K. pneumoniae bioburden in an intensive care unit, as well standardizing the capacity of ICUs (two beds per room) and allocating space for personal belongings, also avoiding storage areas beneath sinks. ${ }^{36}$ Utilizing materials and devices that are easy to sanitize or disinfect will help prevent biofilm formation. Finally, maintaining good quality of water supply in hospitals through appropriate levels of chlorination and checking the quality of water microbiologically using accurate measures are very essential. The negative economic and clinical consequences of the increase of antimicrobial resistance necessitate the prevention of the spread of that resistance. It is associated with high rates of morbidity and mortality. The use of bundles which are evidence-based interventions used for certain patients is recommended in order to enhance patients' outcomes and prevent multidrug resistance.

\section{Conclusions}

$K$. pneumoniae isolates which have been collected in the current study reveal that appropriate measures and important strategies have not been followed. These strategies include monitoring, recording, reporting and evaluating the existence of multidrug resistance. Furthermore, the study showed that infection control guidelines, rapid diagnosis, and quick transmission of date on patient history were not executed by healthcare personnel. More guidance and training should be given to hospital or healthcare personnel on safe decontamination practices, cleaning protocols, avoiding bacterial dissemination and avoiding incorrect dosage. Other important measurements that healthcare providers should follow include antiseptic prevention and therapy of localized infections, toilet use, emptying of urinary bags as well as safe disposal of hospital waste.

The prevention of colonization or infection with MDR organisms is the ideal strategy to be followed by clinicians in order to manage nosocomial infections in ICUs. Clinicians should adopt preventive and treatment strategies as well as infection control to defeat these organisms. Appropriate selection of antibiotic has proven to be effective at minimizing the risks of antibiotic-resistant organisms. However, complete prevention is unrealistic since some patients may be carrying MDR organisms into the ICU at the time when they are admitted to the unit. Therefore, strict compliance to infection control procedures, monitoring cultures of the environment as well as suitable antimicrobial therapy are necessary for the management of these organisms. Therefore, future research should work on developing antimicrobial therapies with activity against MDR organisms through evidence-based infection control procedures so that MDR pathogens could be immediately detected and identified before patients' admission to the ICU. Future studies should address cleaning interventions of inanimate sample sites in ICUs and assess their effectiveness through a prepost sample collection procedure.

\section{References}

1. Kola A, Piening B, Pape UF, et al. An outbreak of carbapenem resistant OXA-48- producing Klebsiella pneumoniae associated to duodenoscopy. Antimicrob Resist Infect Control 2015;4:8

2. Ripabelli G, Tamburro M, Guerrizio G, et al. Tracking multidrug-resistant klebsiella pneumoniae from an italian hospital: molecular epidemiology and surveillance by PFGE, RAPD and PCR-based resistance genes prevalence. Curr Microbiol 2018;9.

3. Kitchel B, Rasheed J, Srinivasan A. Molecular epidemiology of KPC-producing Klebsiella pneumoniae in the United States. In: Abstracts of the forty-eight interscience conference on antimicrobial agents and chemotherapy, Washington, DC, 2008. Abstract C2-3730. American Society for Microbiology, Washington, DC, USA; 2008. p 191.

4. Paterson DL, Bonomo RA. Extended-spectrum beta-lactamases: a clinical update. Clin Microbiol Rev 2005;18:657-86.

5. Bulik CC, Nicolau DP. Double-carbapenem therapy for carbapenemase-producing Klebsiella pneumoniae. Am Agents Chemother 2011;55:3002-4.

6. Hayden MK, Lin M, Lolans K, et al. Centers for Disease Control and Prevention Epicenters Program. Prevention of colonization and infection by Klebsiella pneumoniae carbapenemase-producing enterobacteriaceae in long-term acute-care hospitals. Clin Infect Dis 2015;60:1153-61.

7. Hussein K, Sprecher H, Mashiach T, et al. Carbapenem resistance among Klebsiella pneumoniae isolates: risk factors, molecular characteristics, and susceptibility patterns. Infect. Control Hosp Epidemiol 2009;30:666-71.

8. Aakriti A, Charu J, Sonal S, Ravinder K. Profile of drug resistant gram negative bacteria from ICU at a tertiary care center of India. Asian J Med Health 2017;3:1-7. 
9. Deshpande LM, Jones RN, Fritsche TR, Sader HS. Occurrence and characterization of carbapenemase-producing Enterobacteriaceae: report from the SENTRY antimicrobial surveillance program (2000-2004). Microb Drug Resist 2006;12:223-30.

10. Nordmann P, Cuzon G, Naas T. The real threat of Klebsiella pneumoniaee carbapenemase-producing bacteria. Lancet Infect Dis 2009;9:228-36.

11. Landelle C, Legrand P, Lesprit P, et al. Protracted outbreak of multidrug-resistant Acinetobacter baumannii after intercontinental transfer of colonized patients. Infect Control Hosp Epidemiol 2013;34:119-24.

12. Ikonomidis A, Tokatlidou D, Kristo I, et al. Outbreaks in distinct regions due to a single Klebsiella pneumoniaee clone carrying a blaVIM-1 metallo-b-lactamase gene. J Clin Microbiol 2005;43:5344-7.

13. Livermore DM. The impact of carbapenemases on antimicrobial development and therapy. Curr Opin Investig Drugs 2002;3:218-24.

14. Luzzaro F, Docquier JD, Colinon C, et al. Emergence in Klebsiella pneumoniaee and Enterobacter cloacae clinical isolates of the VIM-4 metallo-lactamase encoded by a conjugative plasmid. Antimicrob Agents Chemother 2004;48:648-50.

15. Oikonomou O, Sarrou S, Papagiannitsis CC, et al. Rapid dissemination of colistin and carbapenem resistant Acinetobacter baumannii in Central Greece: mechanisms of resistance molecular identification and epidemiological data. BMC Infect Dis 2015;15:559.

16. Sambrook J, Fritsch EF, Maniatis T. Molecular cloning: a laboratory manual. New York, NY: Cold Spring Harbor Laboratory Press; 1989.

17. Kumar S, Stecher G, Tamura K. MEGA7: Molecular Evolutionary Genetics Analysis version 7.0 for bigger datasets. Molecul Biol Evol 2016;33:1870-4.

18. Sievert DM, Ricks P, Edwards JR, Schneider A, et al. Antimicrobial-resistant pathogens associated with healthcareassociated infections: summary of data reported to the National Healthcare Safety Network at the Centers for Disease Control and Prevention, 2009-2010. Infect Control Hosp Epidemiol 2013;34:1-14.

19. Sanchez GV, Master RN, Clark RB, et al. Klebsiella pneumoniae antimicrobial drug resistance, United States, 1998-2010. Emerging Infect Dis 2013;19:133.

20. Capone A, Giannella M, Fortini D, et al. High rate of colistin resistance among patients with carbapenem-resistant Klebsiella pneumoniae infection accounts for an excess of mortality. Clin Microbiol Infect 2013;19:E23-30.

21. Guo XL, Wang DC, Zhang YM, et al. Isolation, identification and $16 \mathrm{~S}$ rDNA phylogenetic analysis of Klebsiella pneumonia from diarrhea specimens. Zhonghua Liu Xing Bing Xue Za Zhi 2008;29:1225-9.

22. Fusch C, Pogorzelski D, Main C, et al. Self-disinfecting sink drains reduce the Pseudomonas aeruginosa bioburden in a neonatal intensive care unit. Acta Paediatr 2015;104:344-9.
23. Zheng R, Zhang Q, Guo Y, et al. Outbreak of plasmid-mediated NDM-1-producing Klebsiella pneumoniae ST 105 among neonatal patients in Yunnan, China. Ann Clin Microbiol Antimicrob 2016;15:10.

24. Bures S, Fishbain JT, Uyehara CF, et al. Computer key boards and faucet handles as reservoirs of nosocomial pathogens in the intensive care unit. Am J Infect Control 2000;28:465-71.

25. Resar R, Pronovost P, Haraden C, et al. Using a bundle approach to improve ventilator care processes and reduce ventilator-associated pneumoniae. Jt Comm J Qual Patient Saf 2005;31:243-8

26. Vergara-López S, Domínguez MC, Conejo MC, et al. Wastewater drainage system as an occult reservoir in a protracted clonal outbreak due to metallo- $\beta$ lactamase-producing Klebsiella oxytoca. Clin Microbiol Infect 2013;19:E490-8.

27. Snyder LA, Loman NJ, Faraj LA, et al. Epidemiological investigation of Pseudomonas aeruginosa isolates from a six-yearlong hospital outbreak using high-through put whole genome sequencing. Euro Surveill 2013;18.pii20611.

28. Bhalchandra R, Chandy M, Ramanan VR, et al. Role of water quality assessments in hospital infection control: experience from a new oncology center in eastern India. Indian J Pathol Microbiol 2014;57:435-8.

29. Kramer A, Assadian O. Survival of microorganisms on inanimate surfaces. In: Borkow G, ed. Use of biocidal surfaces for reduction of healthcare acquired infections. Cham: Springer 2014;7-26.

30. Weber DJ, Rutala WA, Kanamori H, et al. Carbapenem-resistant Enterobacteriaceae: frequency of hospital room contamination and survival on various inoculated surfaces. Infect Control Hosp Epidemiol 2015;36:5903.

31. Kramer A, Schwebke I, Kampf G. How long do nosocomial pathogens persist on inanimate surfaces? A systematic review. BMC Infect Dis 2006;6:130.

32. Haverkate MR, Bootsma MC, Weiner S, et al. Modeling spread of KPC-producing bacteria in long-term acute care hospitals in the Chicago region, USA. Infect Control Hosp Epidemiol 2015;36:1148-54.

33. Scotta C, Juan C, Cabot G, et al. Environmental microbiota represents a natural reservoir for dissemination of clinically relevant metallo-beta-lactamases. Antimicrob Agents Chemother 2011;55:5376-9.

34. LaForgia C, Franke J, Hacek DM, et al. Management of a multidrug-resistant Acinetobacter baumannii outbreak in an intensive care unit using novel environmental disinfection: a 38monthreport. Am J Infect Control 2010;38:259-63.

35. Shuman EK, Chenoweth CE. Recognition and prevention of healthcare-associated urinary tract infections in the intensive care unit. Crit Care Med 2010;38:S373-9.

36. Bloomfield S, Exner M, Flemming HC, et al. Lesser-known or hidden reservoirs of infection and implications for adequate prevention strategies: Where to look and what to look for. GMS Hyg Infect Control 2015;10. 